\title{
Enhanced Rashba spin-orbit coupling in core-shell nanowires by the interfacial effect
}

\author{
Paweł Wójcik, 1, a) Andrea Bertoni, ${ }^{2, b)}$ and Guido Goldoni3,2, c) \\ 1) AGH University of Science and Technology, Faculty of Physics and Applied Computer Science, 30-059 Krakow, \\ Al. Mickiewicza 30, Poland \\ 2) S3, Istituto Nanoscienze-CNR, Via Campi 213/a, 41125 Modena, Italy \\ ${ }^{3)}$ Department of Physics, Informatics and Mathematics, University od Modena and Reggio Emilia, \\ Italy
}

(Dated: November 26, 2018)

We report on $\vec{k} \cdot \vec{p}$ calculations of Rashba spin-orbit coupling controlled by external gates in InAs/InAsP core-shell nanowires. We show that charge spilling in the barrier material allows for a stronger symmetry breaking than in homoegenous nano-materials, inducing a specific interface-related contribution to spin-orbit coupling. Our results qualitatively agree with recent experiments [S. Futhemeier et al., Nat. Commun. 7, 12413 (2016)] and suggest additional wavefunction engineering strategies to enhance and control spin-orbit coupling.

Understanding and controlling spin-orbit coupling (SOC) is critical in semiconductor physics. In particular, in semiconductor nanowires (NWs) ${ }^{1}-7$ SOC is essential for to the development of a suitable hardware for topological quantum computation $[9]$, with qubits encoded in zero-mode Majorana states which are supported by hybrid semiconductor-superconductor NWs 10 15. Among other parameters, qubit protection at sufficiently high temperatures relies on a large SOC which determines the topological gap. Additionally, electrical control of SOC is necessary in the realization of spintronic devices 16 26.

SOC arises from the absence of inversion symmetry of the electrostatic potential. In semiconductor NWs, typically having a prismatic shape, finite SOC may be induced by distorting the quantum confinement (Rashba SOC 27) by means of external gates, with the advantage of electrical control. A lattice contribution (Dresselhaus SOC ${ }^{28}$ ) is typically small and may vanish in specific crystallographic directions - for zincblende NWs, the Dresselhaus term vanishes along [111] due to the inversion symmetry.

The Rashba SOC constant $\alpha_{R}$ has been investigated experimentally in homogeneous NWs based on the strong

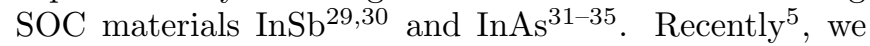
reported on a $\vec{k} \cdot \vec{p}$ approach applied to homogeneous NWs which predicts $\alpha_{R}$ from compositional and structural parameters only. Our calculations performed for InSb NW $s^{5}$ and InAs $\mathrm{NWs}^{\sqrt{36}}$ generally confirm recent experiments in homogeneous NW ${ }^{29131}$, exposing values of $\alpha_{R}$ exceeding by one order of magnitude those reported for $2 \mathrm{D}$ analogous planar systems 37 39. Moreover, $\alpha_{R}$ proved to be strongly tunable with external gates in samples and configurations which can be routinely realized with current technology.

\footnotetext{
a) Electronic mail: pawel.wojcik@fis.agh.edu.pl

b) Electronic mail: andrea.bertoni@nano.cnr.it

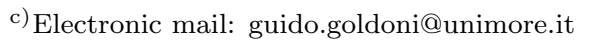

For a quantitative prediction of SOC, it is necessary to take into account valence-to-conduction band coupling, the explicit geometry and crystal structure of the NW, and the electron gas distribution which, in turn, must be self-consistently determined by quantum confinement effects, interaction with dopants and electron-electron interaction. Indeed, in NWs the electron gas localization, and ensuing SOC, is a non-trivial result of competing energy contributions. As a function of doping concentration and ensuing free charge density, the electron gas evolves from a broad cylindrical distribution in the NW core (low density regime) to coupled quasi-1D and quasi-2D channels at the NW edges (large density regime) 40 . Until polygonal symmetry holds, $\alpha_{R}=0$ regardless. However, external gates easily remove the symmetry; again, how $\alpha_{R}$ moves from zero under the influence of the external gates strongly depends on the charge density regime ${ }^{5}$.

In this Letter we extend and apply the $\vec{k} \cdot \vec{p}$ approach to core-shell NWs (CSNWs) and expose a novel mechanism through which SOC can be further tailored, and possibly enhanced. Epitaxially overgrown shells are often used in NW technology, either as a passivating layer improving optical performance ${ }^{44}$, or as a technique to engineer radial heterostructures 45 . Here we show that CSNWs

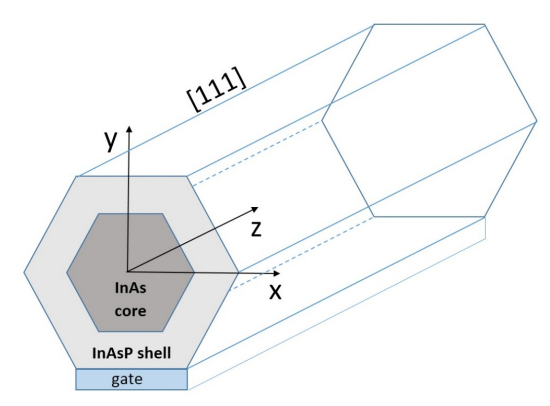

Figure 1. Schematics of a InAs/InAsP CSNW grown along [111] with a bottom gate. 
allow for an increased flexibility in distorting the electron gas of the NWs, giving rise to a specific, interfacial SOC contribution $\underline{46 / 47}$ which substantially increases the total SOC. We make the case for InAs/InAsP CSNWs, a systems of specific interest in photonics $\frac{48}{4}$ and electrical engineering 99 . Our results qualitatively agree with the recent experiments by Furthmeier et al. in Ref. 50 . where the enhancement of SO coupling was measured in GaAs/AlGaAs CSNW, and establish a strategy to increase the SOC in Majorana InAs NWs.

We consider CSNWs with hexagonal cross-section $[51$ grown along [111] (see Fig. 1), assuming in-wire translational invariance along $z$. The used $\vec{k} \cdot \vec{p}$ approach is described in full in Ref. [5, here we focus on generalizations required to account for the contribution of the internal heterointerface. The $8 \times 8$ Kane Hamiltonian is given by $\underline{16}$

$$
H_{8 \times 8}=\left(\begin{array}{cc}
H_{c} & H_{c v} \\
H_{c v}^{\dagger} & H_{v}
\end{array}\right),
$$

where $H_{c}$ and $H_{v}$ are the diagonal matrices corresponding to the conduction $\left(\Gamma_{6 c}\right)$ and valence $\left(\Gamma_{8 v}, \Gamma_{7 v}\right)$ bands whose expressions are given in Ref. 5. Using the perturbative transformation $\mathcal{H}(E)=H_{c}+H_{c v}\left(H_{v}-E\right)^{-1} H_{c v}^{\dagger}$, the Hamiltonian (1) reduces to a $2 \times 2$ effective Hamiltonian for the conduction band electrons. Emphasizing the dependence of material parameters on the position, $\vec{\rho}=(x, y)$,

$$
\begin{aligned}
\mathcal{H}=\left[-\frac{\hbar^{2}}{2} \nabla_{2 D}\right. & \left.\frac{1}{m^{*}(\vec{\rho})} \nabla_{2 D}+\frac{\hbar^{2} k_{z}^{2}}{2 m^{*}(\vec{\rho})}+E_{c}(\vec{\rho})+V(\vec{\rho})\right] \\
& \times \mathbf{1}_{2 \times 2}+\left[\hat{\alpha}_{x}(\vec{\rho}) \sigma_{x}+\hat{\alpha}_{y}(\vec{\rho}) \sigma_{y}\right] k_{z}, \quad(2)
\end{aligned}
$$

where $\sigma_{x(y)}$ are the Pauli matrices and $m^{*}$ is the effective mass given by

$$
\frac{1}{m^{*}(\vec{\rho})}=\frac{1}{m_{0}}+\frac{2 P^{2}}{3 \hbar^{2}}\left(\frac{2}{E_{0}(\vec{\rho})}+\frac{1}{E_{0}(\vec{\rho})+\Delta_{0}(\vec{\rho})}\right),
$$

where $P$ is the conduction-to-valance band coupling parameter.

In Eq. (2), $\hat{\alpha}_{x}, \hat{\alpha}_{y}$ are the SOC operators

$$
\begin{aligned}
\hat{\alpha}_{x} & =\frac{i}{3} P^{2} \hat{k}_{y} \beta(\vec{\rho})-\frac{i}{3} P^{2} \beta(\vec{\rho}) \hat{k}_{y}, \\
\hat{\alpha}_{y} & =\frac{i}{3} P^{2} \hat{k}_{x} \beta(\vec{\rho})-\frac{i}{3} P^{2} \beta(\vec{\rho}) \hat{k}_{x},
\end{aligned}
$$

and $\beta(\vec{\rho})$ is a material-dependent coefficient obtained as follows. In the $i$-th layer

$$
\begin{aligned}
\beta_{i}(\vec{\rho}) & =\frac{1}{E_{c, i}+V(\vec{\rho})-E_{0, i}-E} \\
& -\frac{1}{E_{c, i}+V(\vec{\rho})-E_{0, i}-\Delta_{0, i}-E},
\end{aligned}
$$

where $E_{c}, E_{0}$ and $\Delta_{0}$ are the conduction band edge, the energy gap and the split-off band gap, respectively. Assuming that the above parameters change as a step-like function at the interfaces

$$
\beta(\vec{\rho})=\sum_{i}\left[\beta_{i}(\vec{\rho})-\beta_{i+1}(\vec{\rho})\right] \Omega_{i}(\vec{\rho}),
$$

where the sum is carried out over all the layers, and $\Omega_{i}(\vec{\rho})$ is the shape function, which for the hexagonal section is given by

$$
\begin{aligned}
\Omega_{i}(\vec{\rho}) & =\left[\theta\left(x+x_{i}\right)-\theta\left(x-x_{i}\right)\right]\left[\theta\left(y+y_{i}\right)-\theta\left(y-y_{i}\right)\right] \\
& \times\left[\theta\left(x-y+x_{i}\right)-\theta\left(x-y-x_{i}\right)\right],
\end{aligned}
$$

where $\theta$ is the Heaviside'a function and $\left(x_{i}, y_{i}\right)$ denotes the position of the $(i)$-th interface. Further Taylor expansion gives

$$
\begin{aligned}
\beta_{i}(\vec{\rho}) & \approx\left(\frac{1}{E_{0, i}+\Delta_{0_{i}}}-\frac{1}{E_{0, i}}\right) \\
& +\left(\frac{1}{E_{0, i}^{2}}-\frac{1}{\left(E_{0, i}+\Delta_{0, i}\right)^{2}}\right)\left(E_{c, i}+V(x, y)-E\right) .
\end{aligned}
$$

Substituting (9) into Eqs. (4) and (5), the Rashba coupling constants can be written as

$$
\alpha_{x(y)}(\vec{\rho})=\alpha_{x(y)}^{V}(\vec{\rho})+\alpha_{x(y)}^{i n t}(\vec{\rho}),
$$

i.e., the sum of the SOC induced by the electrostatic potential asymmetry,

$$
\alpha_{x(y)}^{V}(\vec{\rho}) \approx \sum_{i} \frac{1}{3} P^{2}\left(\frac{1}{E_{0, i}^{2}}-\frac{1}{\left(E_{0, i}+\Delta_{0, i}\right)^{2}}\right) \frac{\partial V(\vec{\rho})}{\partial y(x)},
$$

and the interface SOC, related to the electric field at the interfaces between shells,

$$
\alpha_{x(y)}^{i n t}(\vec{\rho}) \approx \sum_{i} \frac{1}{3} P^{2}\left(\tilde{\beta}_{i}-\tilde{\beta}_{i+1}\right) \frac{\partial \Omega_{i}(\vec{\rho})}{\partial y(x)},
$$

with $\tilde{\beta}_{i}=\frac{1}{E_{0, i}+\Delta_{0, i}}-\frac{1}{E_{0, i}}$.

Projecting the 3D Hamiltonian (2) on the basis of inwire states $\psi_{n}(\vec{\rho}) \exp \left(i k_{z} z\right)$, where the envelope functions $\psi_{n}(\vec{\rho})$ are determined by the strong confinement in the lateral direction, leads to SOC matrix elements

$$
\alpha_{x(y)}^{\gamma, n m}=\iint \psi_{n}(\vec{\rho}) \alpha_{x(y)}^{\gamma}(\vec{\rho}) \psi_{m}(\vec{\rho}) d \vec{\rho}
$$

where $\gamma$ identifies the electrostatic $(\gamma=V)$ or the interfacial $(\gamma=i n t)$ contribution.

For the NW in Fig. 1 with a single bottom gate, $\alpha_{y}^{\gamma, n n}=0$ due to inversion symmetry about $y$. Moreover, here we focus on the lowest intra-subband coefficient, $n=1$. Below we discuss the SOC constant $\alpha_{R}=\alpha_{x}^{11}$ and corresponding interfacial and electrostatic components, $\alpha_{R}^{\gamma}=\alpha_{x}^{V, 11}$ and $\alpha_{R}^{\gamma}=\alpha_{x}^{\text {int,11, }}$, respectively.

The electronic states in the CSNW section, $\psi_{n}(\vec{\rho})$, are calculated by a mean-field self-consistent SchödingerPoisson approach 40 . We neglect the exchange-correlation potential which is substantially smaller than the Hartree 


\begin{tabular}{|c|c|c|}
\hline & $\operatorname{InAs}$ & $\operatorname{InAs}_{0.9} \mathrm{P}_{0.1}$ \\
\hline$m^{*}\left[m_{0}\right]$ & 0.0265 & 0.0308 \\
$E_{c}[e V]$ & 0.252 & 0.3 \\
$E_{0}[\mathrm{eV}]$ & 0.42 & 0.5 \\
$\Delta_{0}[\mathrm{eV}]$ & 0.38 & 0.35 \\
\hline
\end{tabular}

Table I. Bulk parameters used in calculations 56 .

potentia $40 \mid 52 / 53$. The gradient of the self-consistent potential $V(\vec{\rho})$ and the corresponding envelope functions $\psi_{n}(\vec{\rho})$ are finally used to determine $\alpha_{R}$ from Eq. (13).

Material parameters mismatch at the interfaces is taken into account solving the eigenproblem $\mathcal{H} \psi_{n}=E \psi_{n}$ with boundary conditions $\underline{46154}$

$$
\begin{aligned}
& \psi_{n}^{(i)}\left(\vec{\rho}_{k}\right)=\psi_{n}^{(j)}\left(\vec{\rho}_{k}\right) \\
& \frac{\hbar^{2}}{2 m^{*(i)}} \nabla_{2 D} \psi_{n}^{(i)}\left(\vec{\rho}_{k}\right)-\frac{\hbar^{2}}{2 m^{*(j)}} \nabla_{2 D} \psi_{n}^{(j)}\left(\vec{\rho}_{k}\right) \\
& +\left[\beta^{(j)}\left(\vec{\rho}_{k}\right)-\beta^{(i)}\left(\vec{\rho}_{k}\right)\right]\left(\sigma_{x}+\sigma_{y}\right) k_{z} \psi_{n}^{(i)}\left(\vec{\rho}_{k}\right)=0,
\end{aligned}
$$

where $\vec{\rho}_{k}$ is the position of the interface between $i$-th and $j$-th shells. Equations (14), (15), depend on both the potential $V(\vec{\rho})$ at the interface and the energy $E$. We eliminate this dependence neglecting the term proportional to $\left(E_{c, i}+V(\vec{\rho})-E\right)$ in the Taylor series, Eq. (9). Then, the interface contributions 12 are determined fully by material parameters. This assumption, justified when $\beta^{(j)}-\beta^{(i)}$ is small, neglects the SOC related to the motion of electron in the $\vec{\rho}$ plane, which in general contributes to the SOC by the boundary conditions.

Below we investigate a InAs $50 \mathrm{~nm}$-wide core (measured facet-to-facet) surrounded by a $30 \mathrm{~nm} \mathrm{InAs} \mathrm{I}_{1-x} \mathrm{P}_{x}$ shell, with $x=0.1$ which allows to neglect strain-induced SOC 55 Furthermore, as shown below, interfacial SOC is enhanced by the easy penetration of envelope functions in low band offset barriers, here only $48 \mathrm{meV}$ high. Simulations have been carried out for a temperature $T=4.2 \mathrm{~K}$, in the constant electron concentration regime. The parameters adopted are given in Tab. I. $P$ is assumed to be constant throughout the materials and $E_{P}(\operatorname{InAs})=2 m_{0} P^{2} / \hbar^{2}=21.5 \mathrm{eV}$.

The calculated SOC coefficients for the InAs/InAsP CSNW of Fig. 1 as a function of the back gate voltage is reported in Fig. 2(a). The SOC constant is trivially zero if $V_{g}=0$, due to the overall inversion symmetry. At any finite voltage the inversion symmetry is removed, hence $\alpha_{R} \neq 0$. As shown in Fig. 22(a), the total $\alpha_{R}$ ensues from two different contributons, namely interfacial and electrostatic, whose magnitude is of the same order. It is thus crucial to include both of them in the assessment of SOC in CSNWs. Note that the electrostatic component almost coincides with the value for an InAs NW with the same geometry, but no overgrown shel[57. However, for this specific nanostructure, the largest part of $\alpha_{R}$ is due to the interfacial contribution, which is $\approx 50 \%$ larger than the electrostatic one. While the ratio between the two contributions is nearly independent of $V_{g}$ [see the in-

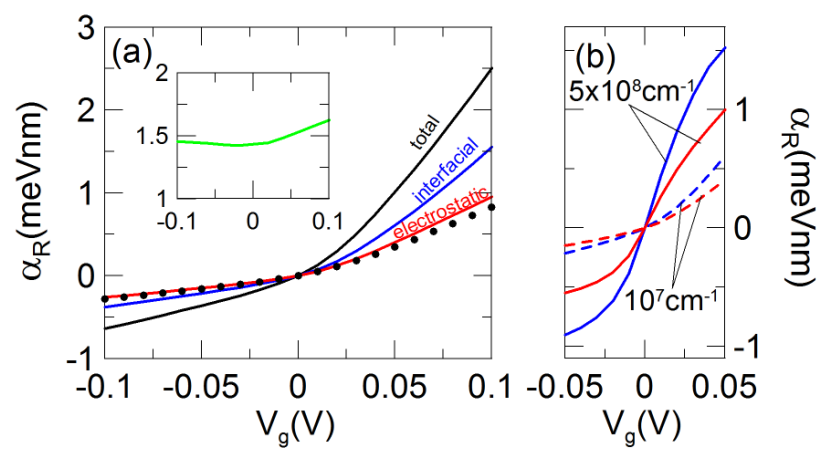

Figure 2. (a) Lines: total, electrostatic and interfacial SOC constants vs gate voltage $V_{g}$, according to labels. Dots: total SOC constant for an equivalent homegeneous InAs NW. Inset: ratio between interfacial and electrostatic components, $\alpha_{R}^{i n t} / \alpha_{R}^{V}$. Results for $n_{e}=10^{7} \mathrm{~cm}^{-1}$. See text for structure and material parameters. (b) Electrostatic and interfacial SOC constants vs $V_{g}$ around $V_{g}=0$ showing shooting up of SO couplings for higher electron density.

set in Fig. 2(a)], they are both strongly anisotropic with respect to the field direction. This is due to the different effects on the charge density, as discussed in Ref. 5. This effect can also be grasped from the probability distribution reported in Fig. 3 (top and bottom rows). Indeed, the positive $V_{g}$ pushes electron states towards the interface opposite to the gate, where the gradient of the self-consistent field is low. On the other hand, at $V_{g}<0$ electrons are pulled to the region of the nearest interface with the stronger electric field, additionally strengthened by the electron-electron interaction ${ }^{5}$.
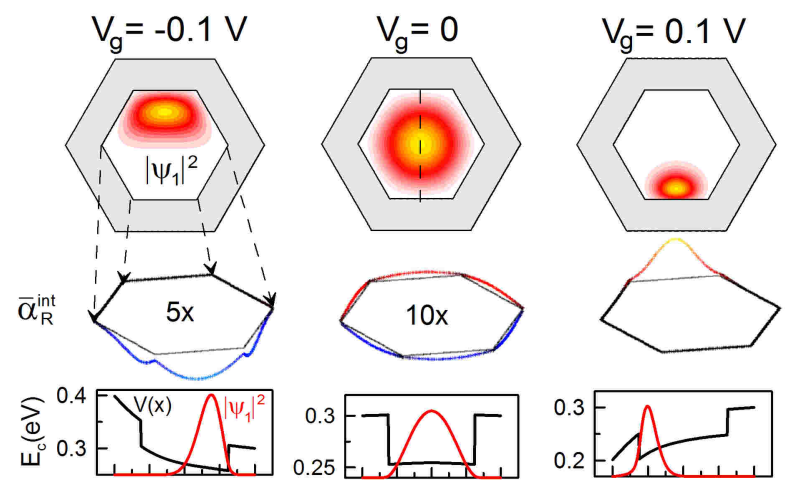

Figure 3. Top row: Square of the ground state envelope function $\left|\psi_{1}(x, y)\right|^{2}$. Middle row: linear density of the interfacial SOC constants at interfaces. Bottom row: self-consistent potential profile (black line) and $\left|\psi_{1}(x, y)\right|^{2}$ (red line) along the facet-to-facet dashed line marked in the top-middle panel. Results at selected gate voltages $V_{g}=-0.1,0,0.1 \mathrm{~V}$ for the same structure as in Fig. 2.

The value of $\alpha_{R}^{i n t}$ depends on the penetration of the 
wave function into the interfaces. As shown in Fig. 3 (middle row) the linear density of interfacial SOC at the interfaces $\bar{\alpha}_{R}^{i n t}=\psi_{1}(\vec{\rho}) \alpha_{R}^{i n t}(\vec{\rho}) \psi_{1}(\vec{\rho})$ is finite almost everywhere, but it has opposite sign at opposite facets $\underline{58}$ For a centro-symmetric system $\left(V_{g}=0\right)$ the overall value is zero, since opposite contributions cancel out exactly. We stress a remarkable difference between CSNWs and analogous planar structures. In a planar asymmetric quantum well, for example, $\alpha_{R} \neq 0$. In a CSNW with an embedded quantum well, however, the overall symmetry is recovered even if each facet of the quantum well is individually asymmetric. Therefore, opposite segments have opposite Rashba contributions and compensate. However, any asymmetric gate potential unbalances opposite contributions, the total effect being related to the amount of envelope function at the interface.

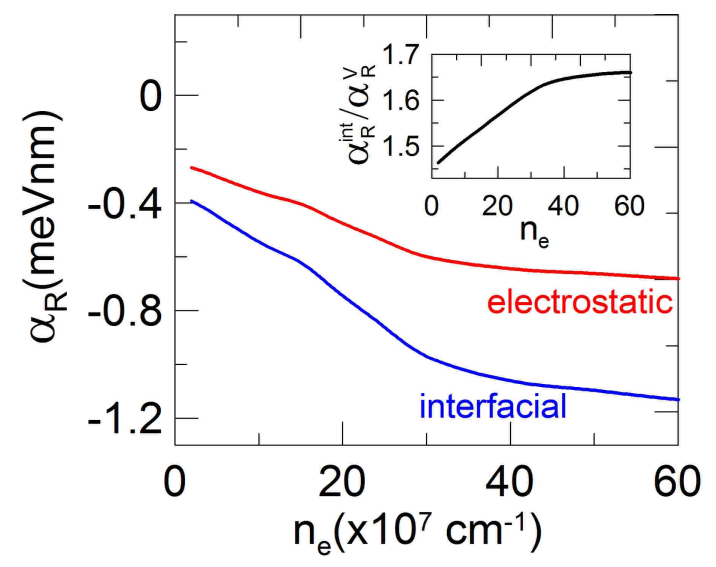

Figure 4. Electrostatic $\alpha_{R}^{V}$ and interfacial $\alpha_{R}^{i n t}$ contributions of SOC constant $v s$ electron density $n_{e}$. Results for $V_{g}=$ $-0.1 \mathrm{~V}$. Inset: ratio $\alpha_{R}^{i n t} / \alpha_{R}^{V}$ vs $n_{e}$.

Note the almost linear increase of $\alpha_{R}$ with $V_{g}$. This behaviour is observed in a relatively small charge density regime: the average Coulomb energy is small, most of the charge is located in the core, and it is relatively rigid to an applied transverse electric field. At larger densities, however, charge moves at the interfaces to minimize

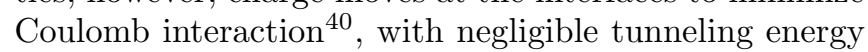
between opposite facets. In this regime, the symmetric charge density distribution is unstable and it is easily distorted by an electric field ${ }^{5}$. Accordingly, SOC constant shoots around $V_{g}=0$ as soon as the gate is switched on - see Fig. 2(b).

As we show in Fig. 4 both SOC components substantially increase in intensity with charge density, while their ratio is weakly affected, it being rather a property of the nano-material (band parameters and band offset). This is explicitly shown in Fig. 5. where the two contributions are plot $v s$ the stechiometric fraction $x$. At low $x$, penetration is very large, and the interfacial effect is dominant (of course for $x=0$ the heterostructure is an homogeneous NW with a larger diameter), while as $x=0.15$ the two contributions are comparable, as also shown in the inset.

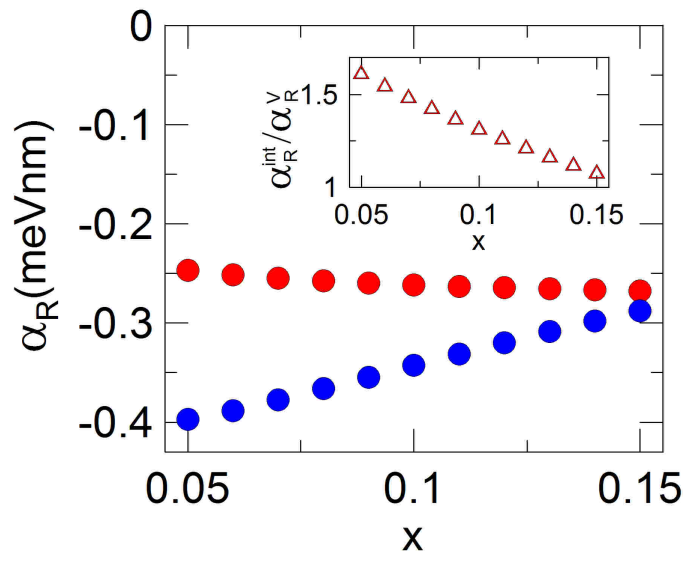

Figure 5. The interfacial $\alpha_{R}^{\text {int }}$ (blue circles) and electrostatic $\alpha_{R}^{V}$ (red circles) SOC constants vs $\operatorname{InAs}_{1-x} \mathrm{P}_{x}$ alloy composition, $x$. Inset: $\alpha_{R}^{\text {int }} / \alpha_{R}^{V}$ vs $x$. Results for $V_{g}=-0.1 \mathrm{~V}$ and $n_{e}=10^{7} \mathrm{~cm}^{-1}$.

To summarize, we have shown that Rashba SOC in CSNWs is increased by the effect of the radial heterointerface, and its control via external metallic gates may be highly improved by this interfacial effect. Although we did not attempt to optimize $\alpha_{R}$ in the many parameter space allowed by CSNWs, our results suggest that a general strategy to enhance SOC in CSNWs relies on a modification of the compositional structure exploiting asymmetric penetration of the wave function into the shell layer.

This work was partially supported by the AGH UST statutory tasks No. 11.11.220.01/2 within subsidy of the Ministry of Science and Higher Education and in part by PL-Grid Infrastructure. P.W. was supported by National Science Centre, Poland (NCN) according to decision 2017/26/D/ST3/00109.

\section{REFERENCES}

${ }^{1}$ I. A. Kokurin, Physica E 74, 264 (2015).

${ }^{2}$ I. A. Kokurin, Solid State. Commun. 195, 49 (2014).

${ }^{3}$ M. Kammermeier, P. Wenk, J. Schliemann, S. Heedt, and T. Schäpers, Phys. Rev. B 93, 205306 (2016)

${ }^{4}$ G. W. Winkler, D. Varjas, R. Skolasinski, A. A. Soluyanov, M. Troyer, and M. Wimmer, Phys. Rev. Lett. 119, 037701 (2017)

${ }^{5}$ P. Wójcik, A. Bertoni, and G. Goldoni, Phys. Rev. B 97, 165401 (2018)

${ }^{\circ}$ T. Campos, P. E. Faria Junior, M. Gmitra, G. M. Sipahi, and J. Fabian, Phys. Rev. B 97, 245402 (2018)

${ }^{7}$ J.-W. Luo, S.-S. Li, and A. Zunger, Phys. Rev. Lett. 119, 126401 (2017)

${ }^{\curlyvee}$ J. Alicea, Phys. Rev. B 81, 125318 (2010)

${ }^{9}$ J. Sau, Physics 10, 68 (2017).

${ }^{10}$ A. Kitaev, Ann. Phys. 303, 2 (2003).

${ }^{11}$ J. D. Sau, S. Tewari, and S. Das Sarma, Phys. Rev. B 85, 1 (2012). 
${ }^{12}$ V. Mourik, K. Zuo, S. M. Frolov, S. R. Plissard, E. P. A. M. Bakkers, and L. P. Kouwenhoven, Science 336, 1003 (2012).

${ }^{13}$ J. Mastomäki, S. Roddaro, M. Rocci, V. Zannier, D. Ercolani, L. Sorba, I. J. Maasilta, N. Ligato, A. Fornieri, E. Strambini, and F. Giazotto, Nano Research 10, 3468 (2017)

${ }^{14}$ S. M. Albrecht, A. P. Higginbotham, M. Madsen, F. Kuemmeth, T. S. Jespersen, J. Nygåard, P. Krogstrup, and C. M. Marcus, Nature 531, 206 (2016).

${ }^{15}$ A. Manolescu, A. Sitek, J. Osca, L. m. c. Serra, V. Gudmundsson, and T. D. Stanescu, Phys. Rev. B 96, 125435 (2017).

${ }^{16}$ J. Fabian, A. Matos-Abiague, C. Ertler, P. Stano, and I. Žutić, Acta Physica Slovaca 57, 565 (2007).

${ }^{17}$ S. Datta and B. Das, Appl. Phys. Lett. 56, 665 (1990).

${ }^{18}$ J. Schliemann, J. C. Egues, and D. Loss, Phys. Rev. Lett. 90, 146801 (2003).

${ }^{19}$ P. Wójcik and J. Adamowski, Semicond. Sci. Technol. 31, 115012 (2016).

${ }^{20} \mathrm{P}$. Wójcik, J. Adamowski, B. J. Spisak, and M. Wołoszyn, J. Appl. Phys. 115, 104310 (2014).

${ }^{21}$ P. Wójcik and J. Adamowski, Sci Rep 7, 45346 (2017).

${ }^{22}$ P. P. Das, J. Bhandari, N abd Wan, M. Cahay, K. B. Chetry, R. S. Newrock, and S. T. Herbert, Nanotechnology 23, 215201 (2012).

${ }^{23}$ P. Debray, S. Rahman, and M. Muhammad, Nature Nano Technol. 4, 759 (2009).

${ }^{24}$ M. Kohda, S. Nakamura, Y. Nishihara, K. Kobayashi, T. Ono, J. O. Ohe, Y. Tokura, T. Mineno, and J. Nitta, Nature Communications 3, 1082 (2012)

${ }^{25}$ F. Rossella, A. Bertoni, D. Ercolani, M. Rontani, L. Sorba, F. Beltram, and S. Roddaro, Nat. Nanotechnol. 9, 997 (2014)

${ }^{26}$ A. Iorio, M. Rocci, L. Bours, M. Carrega, V. Zannier, L. Sorba, S. Roddaro, F. Giazotto, and E. Strambini, ArXiv e-prints (2018), arXiv:1807.04344 [cond-mat.mes-hall].

${ }^{27}$ E. I. Rashba, Sov. Phys. Solid State 2, 1109 (1960).

${ }^{28}$ G. Dresselhaus, Phys. Rev. 100, 580 (1955).

${ }^{29}$ I. van Weperen, B. Tarasinski, D. Eeltink, V. S. Pribiag, S. R. Plissard, E. P. A. M. Bakkers, L. P. Kouwenhoven, and M. Wimmer, Phys. Rev. B 91, 201413(R) (2015).

${ }^{30}$ J. Kammhuber, M. C. Cassidy, F. Pei, M. P. Nowak, A. Vuik, D. Car, S. R. Plissard, E. P. A. M. Bakkers, M. Wimmer, and L. P. Kouwenhoven, Nat Commun. 8, 478 (2017).

${ }^{31}$ Z. Scherübl, G. m. H. Fülöp, M. H. Madsen, J. Nygård, and S. Csonka, Phys. Rev. B 94, 035444 (2016).

${ }^{32}$ A. E. Hansen, M. T. Björk, C. Fasth, C. Thelander, and L. Samuelson, Phys. Rev. B 71, 205328 (2005)

${ }^{33}$ S. Dhara, H. S. Solanki, V. Singh, A. Narayanan, P. Chaudhari, M. Gokhale, A. Bhattacharya, and M. M. Deshmukh, Phys. Rev. B 79, 121311 (2009)

${ }^{34}$ P. Roulleau, T. Choi, S. Riedi, T. Heinzel, I. Shorubalko, T. Ihn, and K. Ensslin, Phys. Rev. B 81, 155449 (2010)

${ }^{35}$ S. Estévez Hernández, M. Akabori, K. Sladek, C. Volk, S. Alagha, H. Hardtdegen, M. G. Pala, N. Demarina, D. Grützmacher, and T. Schäpers, Phys. Rev. B 82, 235303 (2010)

${ }^{36} \mathrm{P}$. Wójcik, A. Bertoni, and G. Goldoni, (2018), unpublished.

${ }^{37}$ R. L. Kallaher, J. J. Heremans, N. Goel, S. J. Chung, and M. B. Santos, Phys. Rev. B 81, 0035335 (2010).

${ }^{38}$ R. L. Kallaher, J. J. Heremans, N. Goel, S. J. Chung, and M. B. Santos, Phys. Rev. B 81, 075303 (2010).

${ }^{39}$ F. Herling, C. Morrison, C. S. Knox, S. Zhang, O. Newell, M. Myronov, E. H. Linfield, and C. H. Marrows, Phys. Rev. B 95, $155307(2017)$
${ }^{40}$ A. Bertoni, M. Royo, F. Mahawish, and G. Goldoni, Phys. Rev. B 84, 205323 (2011)

${ }^{41}$ S. Funk, M. Royo, I. Zardo, D. Rudolph, S. Morktter, B. Mayer, J. Becker, A. Bechtold, S. Matich, M. Dblinger, M. Bichler, G. Koblmller, J. J. Finley, A. Bertoni, G. Goldoni, and G. Abstreiter, Nano Letters 13, 6189 (2013)

42 J. Jadczak, P. Plochocka, A. Mitioglu, I. Breslavetz, M. Royo, A. Bertoni, G. Goldoni, T. Smolenski, P. Kossacki, A. Kretinin, H. Shtrikman, and D. K. Maude, Nano Letters 14, 2807 (2014)

${ }^{43}$ M. Royo, A. Bertoni, and G. Goldoni, Phys. Rev. B 89, 155416 (2014)

${ }^{44}$ F. Jabeen, S. Rubini, V. Grillo, L. Felisari, and F. Martelli, Appl. Phys. Lett. 93, 083117 (2008).

${ }^{45}$ D. Spirkoska, J. Arbiol, A. Gustafsson, S. Conesa-Boj, F. Glas, I. Zardo, M. Heigoldt, M. H. Gass, A. L. Bleloch, S. Estrade, M. Kaniber, J. Rossler, F. Peiro, J. R. Morante, G. Abstreiter, L. Samuelson, and A. Fontcuberta i Morral, Phys. Rev. B 80 , 245325 (2009)

${ }^{46}$ Z. A. Devizorova and V. A. Volkov, JETP Lett. 98, 101 (2013).

${ }^{47}$ E. L. Ivchenko, A. Y. Kaminski, and U. Rössler, Phys. Rev. B 54, 5852 (1996).

${ }^{48}$ J. Treu, M. Bormann, H. Schmeiduch, M. Dblinger, S. Morktter, S. Matich, P. Wiecha, K. Saller, B. Mayer, M. Bichler, M.-C. Amann, J. J. Finley, G. Abstreiter, and G. Koblmller, Nano Letters 13, 6070 (2013)

${ }^{4 y}$ X. Liu, P. Liu, H. Huang, C. Chen, T. Jin, Y. Zhang, X. Huang, Z. Jin, X. Li, and Z. Tang, Nanotechnology 24, 245306 (2013).

${ }^{50}$ S. Furthmeier, F. Dirnberger, M. Gmitra, A. Bayer, M. Forsch, J. Hubmann, C. Schüller, E. Reiger, J. Fabian, T. Korn, and D. Bougeard, Nat Commun. 7, 12413 (2016).

${ }^{51}$ A. Sitek, M. Urbaneja Torres, K. Torfason, V. Gudmundsson, A. Bertoni, and A. Manolescu, Nano Letters 18, 2581 (2018).

${ }^{52}$ B. M. Wong, F. Lonard, Q. Li, and G. T. Wang, Nano Letters 11, 3074 (2011).

${ }^{53}$ M. Royo, C. Segarra, A. Bertoni, G. Goldoni, and J. Planelles, Phys. Rev. B 91, 115440 (2015)

${ }^{54}$ E. A. de Andrada e Silva, G. C. La Rocca, and F. Bassani, Phys. Rev. B 55, 16293 (1997)

${ }^{55}$ Lattice mismatch and strain field are additional sources of SOC, which are neglected for the present lattice matched heterostructures. We also neglect a SOC contribution resulting from the interface inversion asymmetry related to different atomic termination at opposite interface ${ }^{59 / 60}$ which is small. Moreover, additional calculations with slight smearing of the potential at the interface instead of the step like function did not lead to significantly different results.

${ }^{56}$ I. Vurgaftman, J. R. Meyer, and L. R. Ram-Mohan, J. Appl. Phys. 89, 5815 (2001).

${ }^{57}$ For the model CSNW under consideration, the gate is attached directly to the NW, so that $\alpha_{R}$ is an upper bound value, while any dielectric interlayer would decrease $\alpha_{R}$. For the NW with no shell, the gate has been placed at the same distance from the core as in the case with the shell, in other to compare the two calculations.

${ }^{58}$ The sign of the SOC linear density on each facet is decided by the choice of the reference frame. Since $\hat{\alpha}_{x}$ appears combined with the spin matrices in the Hamiltonian (2), the overall sign of the SOC term is unaffected by a change in the reference frame.

${ }^{59}$ E. L. Ivchenko, A. Y. Kaminski, and U. Rössler, Phys. Rev. B 54, $5852(1996)$

${ }^{60}$ U. Rössler and J. Kainz, Solid State Commun. 121, 313 (2002). 